\title{
Auslösung von Blütenbildung bei Langtagpflanzen unter Kurztagbedingungen durch Aufpfropfung von Kurztagpflanzen
}

\author{
Von Anton Lang und Georg Melchers \\ Aus dem Kaiser-Wilhelm-Institut für Biologie, Tübingen, früher Berlin-Dahlem \\ (Z. Naturforschg. 3b, 108-111 [1948]; eingegangen am 19. März 1948)
}

\begin{abstract}
In Kurztag gehaltene Pflanzen von Hyoscyamus niger (einjährig) werden durch Aufpfropfung von Maryland-Mammut-Tabak-Reisern zur Blütenbildung gebracht, wenn die Spender sich unter Kurztagbedingungen befinden. Wurden die Spender unter Langtagbedingungen gehalten, so wurde Blütenbildung an den Empfängern nur ausnahmsweise und nur an nachträglich austreibenden Seitensprossen beobachtet. An in Kurztag gehaltenen Nicotiana-silvestris-Pflanzen rief Aufpfropfung von Maryland-Mammut-Blättern in einigen Fällen Blütenbildung hervor, gleichgültig, ob die Reiser unter Kurztagoder Langtagbedingungen gehalten wurden; es ist aber fraglich, ob es sich um eine spezifische Wirkung des Reizes auf die Blütenbildung oder einen allgemeineren, ernährungsphysiologischen Einfluß handelt. Der für die Auslösung der Blütenbildung maßgebende „Endzustand“ ist bei Lang- und Kurztagpflanzen derselbe.
\end{abstract}

$\mathrm{I}$

n den zur Auslösung von Blütenbildung vorgenommenen Pfropfversuchen mit tageslängenabhängigen Pflanzen dienten aus technischen Gründen als nicht-blühfähige Partner („Empfänger") durchweg Kurztagplanzen in Langtagbedingungen, als blühfähige („Spender“) fast immer entweder induzierte Exemplare derselben Art oder tagneutrale Pflanzen ${ }^{1-4}$. Nur M elchers und Lang ${ }^{5}$ verwendeten als Spender auch ausgesprochene (qualitativ reagierende) Langtagpflanzen, nämlich Hyoscyamus niger (einjährige Rasse) und Nicotiana silvestris, die, auf die Kurztagpflanze Nicotiana tabacum ,MarylandMammut" gepfropft, sich hinsichtlich Auslösung der Blütenbildung als noch etwas wirksamer erwiesen als tagneutrale Formen (Nicotiana tabacum ,Java“ und „Cavalla“).

Wir berichten im folgenden zur Ergänzung über einige Pfropfversuche, in denen unter Kurztagbedingungen gehaltene Langtagplanzen als Empfänger und Kurztagpflanzen als Spender verwendet wurden. Diese Versuche wurden in den Jahren 1942 und 1943 im Rahmen eines größeren Programms ausgeführt, in welchem die physiologischen Beziehungen der beiden photoperiodischen Reaktionstypen genauer untersucht werden sollten. Die Auslösung von Blütenbildung an

1 M. H. C a jl a h j a n, C.R. Acad. Sci. URSS., N.S. 13, 77 [1936].

${ }^{2}$ J. K u i jpe r u. L. K. Wi e r sum, Proc., Kon. nederl. Akad. Wetensch. 39, 3 [1936].

${ }^{3}$ B. S. Mo šk ov, Bull. appl. Bot., Genet. and Plant-Breed., Ser. A, Nr. 21, 145 [1937]. nicht-blühfähigen photoperiodisch empfindlichen Pflanzen durch blühfähige wird gewöhnlich als Wirkung des aus dem Spender in den Empfänger übertretenden "Blühhormons“ (oder mehrerer „Blühhormone") gedeutet. Gleichgültig, ob man das für erwiesen hält oder nicht, ist es sicher, daß die Erreichung des von einer Pflanze auf die andere übertragbaren „Zustandes“ der Blühfähigkeit von der Tageslänge abhängt. Der Vorgang, an dem die Tageslänge eingreift, braucht bei den Lang- und Kurztagpflanzen jedoch nicht derselbe zu sein; ja, da bei den beiden Reaktionstypen dieselbe Bedingung, Langtag oder Kurztag, gegensätzlich wirkt, ist das sogar unwahrscheinlich. Dann kann es aber $u$. U. möglich sein, die Blütenbildung durch einen selbst nicht blühfähigen Pfropfpartner auszulösen, wie das aus Pfropfungen zwischen nicht kältebehandelten zweijährigen Pflanzen und Kurztagpflanzen bereits bekannt ist $^{6}$. Die äußeren Umstände haben die Durchführung des vorgesehenen Programms verhindert. Da seine Wiederaufnahme vorerst noch ungewiß ist, da wir andererseits aber die bereits erzielten Ergebnisse schon in verschiedenen Veröffentlichungen verwertet haben ${ }^{7,8}$, scheint es uns angebracht, das Versuchsmaterial im einzelnen vorzulegen.

${ }^{4}$ K. C. H a mne r u. J. B on ne r, Bot. Gaz. 100, 388 [1938].

5 G. M e l chers u. A. Lang, Biol. Zbl. 61, 16 [1941].

${ }^{6}$ G. Melchers, Ber. dtsch. bot. Ges. 57, 29 [1939].

7 A. Lang u. G. Melchers, Planta 33, 653 [1943].

8 G. Melchers u. A. Lang, Biol. Zbl. 67, 105 [1948]. 


\section{Material, Methodisches}

Als Langtagpflanzen dienten wiederum Hyoscyamus niger (einjährige gelbblühende Rasse) und Nicotiana silvestris, als Kurztagpflanze Maryland-Mammut-Tabak (vgl. Anm. 5). Bis zur Herstellung der Pfropfung befanden sich beide Partner in der jeweils nichtinduktiven Tageslänge. Die Langtagpflanzen wurden durchweg als Unterlagen, die Kurztagpflanze als Reis verwendet. Die Hyoscyamus-Pflanzen wurden zur Pfropfung, wie früher beschrieben ${ }^{\mathbf{9}}$, derart eingetopft, daß ein Teil der Rübe über die Erde und den Topfrand herausragte. Die Rosettenachse von Nicotiana silvestris befand sich über der Erde, hier wurde nur der Erdballen etwas höher gesetzt. Wenn ganze Pflanzen von Maryland-Mammut zur Pfropfung verwendet wurden, so wurden sie, um eine Blütenbildung am Reis selbst zu verhindern, nach der Pfropfung, meist wenn etwa 10 größere Blätter vorhanden waren, dekapitiert, und alle Achselknospen wurden entfernt. Sollte das Reis induziert werden, so wurde die ganze Pfropfung mit Hilfe von Pappstürzen unter Kurztagbedingungen gehalten. Sollte es unter Langtagbedingungen bleiben, so fanden zur Kurztaghaltung der Unterlage Überzüge aus lichtundurchlässigem Stoff (außen gegen übermäßige Wärmeabsorption weiß benäht, verschließbar mit Hilfe von einfachen Klammern) Verwendung (Abb. 1).* Bei Abbruch eines Versuches wurden alle Pflanzen, die keine sichtbaren Blütenanlagen hervorgebracht hatten, unter dem Präpariermikroskop auf das etwaige Vorhandensein von solchen untersucht.

\section{Versuche}

a) Hyoscyamus niger als Empfänger.

Mit Hyoscyamus als Empfänger wurde 1942 und 1943 je eine größere Versuchsreihe ausgeführt. Da einjährige Hyoscyamus-niger-Pflanzen bei Entblätterung unabhängig von der Tageslänge Blüten anlegen ${ }^{7,10}$, konnte die bei zweijährigen ausgearbeitete Methode der Freilegung der Vegetationsspitze $^{9}$ nicht verwendet werden, und der Pfropfschnitt war durch die Blattrosette, möglichst genau neben den Vegetationspunkt, zu führen; jedoch wurden die älteren Blätter vor der Pfropfung und weiterhin fortlaufend nach Maßgabe des Zuwachses entfernt. Das Ergebnis des Versuches von 1942 zeigt Tab. 1.

Von den ganz in Kurztag gehaltenen Pfropfungen kam in 24 Fällen von 28 auswertbaren der Empfänger zur Blütenbildung; von denen, bei welchen sich das Reis in Langtag befand, also unter den nicht-induktiven Tageslängenbedingungen, in 2 Fällen von 26. In diesen beiden Fällen

* Abb. 1 u. 2 s. Tafel, S. 92 b.

9 G. Melchers, Biol. Zbl. 57, 568 [1937].

10 A. L an g, Biol. Zbl. 61, 427 [1941].

\begin{tabular}{|c|c|c|c|c|c|}
\hline & \multicolumn{2}{|c|}{ Zahl der Pfropfungen } & \multicolumn{2}{c|}{ Ergebnis } \\
\cline { 2 - 5 } Behandlungsweise & $\begin{array}{c}\text { herge- } \\
\text { stellt }\end{array}$ & $\begin{array}{c}\text { ausge- } \\
\text { fallen }\end{array}$ & $\begin{array}{c}\text { aus- } \\
\text { wertbar }\end{array}$ & $\begin{array}{c}\text { E mit } \\
\text { Bliuten- } \\
\text { anlagen }\end{array}$ & $\begin{array}{c}\text { E } \\
\text { vege- } \\
\text { tativ }\end{array}$ \\
\hline E in Kurztag, & & & & & \\
S in natürl. Tag & 30 & 4 & $\mathbf{2 6}$ & $2^{*}$ & $\mathbf{2 4}$ \\
E u. S in Kurztag & 30 & 2 & $\mathbf{2 8}$ & $\mathbf{2 4}^{* *}$ & 4 \\
\hline
\end{tabular}

* Blütenbildung an Seitentrieben, Haupttrieb vegetativ.

** In 11 Fällen Blütenbildung auch am Haupttrieb, in 13 nur an Seitentrieben.

Tab.1. Pfropfung von Nicotiana tabacum MarylandMammut als Spender (S) auf Hyoscyamus niger einjährig als Empfänger (E). Versuche von 1942.

Protokoll-Nr. 1942/I. Aussaat: Hyoscyamus 8.1. (Kultur bis 24. 2. in nat. Tag, dann in $~ 10$-stdg. Kurztag), Maryland-Mammut 8.1. und 15.3. (Kultur bis 1.4. mit Zusatzlicht von Dämmerungsbeginn bis $0^{\circ 0}$ Uhr, dann in nat. Tag). Herstellung der Pfropfungen 25.5. und 9. bis 10.6., Beobachtungsdauer 100 Tage. Kurztag: $\sim 10$ Stdn. Licht täglich $\left(\sim 7^{00}\right.$ bis $\left.17^{00} \mathrm{Uhr}\right)$.

wurden Blüten von Seitensprossen gebildet, die nachträglich im entblätterten Teil der Rübe austrieben, während der Haupt-(Primär-)trieb strikt vegetativ blieb. Wir halten es daher für wahrscheinlich, daß die Auslösung der Blütenbildung hier nicht auf den Einfluß des Reises zurückzuführen ist, sondern darauf, daß diese Vegetationspunkte dem hemmenden Einfluß der Blätter auf die Blütenbildung ${ }^{7,10}$ entzogen blieben. Allerdings sind dann auch bei den Pfropfungen mit induzierten Reisern diejenigen, bei denen die Blütenbildung auf Seitentriebe beschränkt blieb, insgesamt 13. Exemplare, von der Gesamtzahl der zur Blütenbildung gekommenen abzusetzen; die Zahl der Pfropfungen, bei denen die Blütenbildung eindeutig unter dem Einfluß des Reises zustande kam, reduziert sich damit von 28 auf 11. Ein Einfluß der blühfähigen Reiser gegenüber den nicht blühfähigen ist auf jeden Fall unverkennbar (vgl. Abb. 2).

Im Jahre 1943 wurden unter Berücksichtigung dieser Erfahrungen bei den sonst in gleicher Weise hergestellten Pfropfungen alle nachträglich austreibenden Seitenvegetationspunkte des Empfängers entfernt. Außerdem wurden die Empfänger stärker entblättert, so daß sie fortlaufend nur $1-2$ voll entfaltete Blätter besaßen. Bei vernalisierten zweijährigen Hyoscyamus-Pflanzen wird unter Kurztagbedingungen Blütenbildung bereits durch kleinere Blätter verhindert?. Zur Kontrolle wurden überdies ebenso behandelte, 


\begin{tabular}{|c|c|c|c|c|c|}
\hline & \multicolumn{2}{|c|}{ Zahl der Pfropfungen } & \multicolumn{2}{|c|}{ Ergebnis } \\
\cline { 2 - 5 } Behandlungsweise & $\begin{array}{c}\text { berge- } \\
\text { stellt }\end{array}$ & $\begin{array}{c}\text { ausge- } \\
\text { fallen }\end{array}$ & $\begin{array}{c}\text { aus- } \\
\text { wertbar }\end{array}$ & $\begin{array}{c}\text { E mit } \\
\text { Blüten- } \\
\text { anlagen }\end{array}$ & $\begin{array}{c}\text { E } \\
\text { vege- } \\
\text { tativ }\end{array}$ \\
\hline E in Kurztag, & & & & & \\
S in natürl. Tag & 25 & 7 & $\mathbf{1 8}$ & - & $\mathbf{1 8}$ \\
E u. S in Kurztag & 25 & 4 & $\mathbf{2 1}$ & $\mathbf{2 0}$ & - \\
Hyoscyamus, teil- & & & & & \\
entblättert, in na- \\
türl. Tag. . . . & 10 & 4 & $\mathbf{6}^{*}$ & $\mathbf{6}^{*}$ & - \\
desgl. in Kurztag & 10 & - & $10^{* *}$ & - & $10^{* *}$ \\
\hline
\end{tabular}

* Mittlere Lebensdauer bis zum Schossen 33 Tage.

** Lebensdauer 25-45, im Mittel 33 Tage.

Tab. 2. Pfropfungen zwischen Maryland-Mammut (Spender, S) und Hyoscyamus niger (Empfänger, E). Versuche von 1943.

Protokoll-Nr. 1943/I. Aussaat: 15.1.; Hyoscyamus ab 21. 2. in Kurztag, Maryland-Mammut bis 1.3. mit $\mathrm{Zu}$ satzlicht bis $0^{00}$ und ab $7^{00}$ (vom Beginn bzw. bis zum Ende der Dämmerung). Herstellung der Pfropfungen 29.6. bis 2.7., Beobachtungsdauer 72 Tage. Kurztag: Licht $7^{15}$ bis $16^{30}$ Uhr täglich. Unterlagen (E) laufend bis auf $1-2$ entfaltete Blätter entblättert; alle Seitentriebe entfernt.

aber ungepfropfte einjährige Hyoscyamus-Exemplare in Kurz- wie Langtagbedingungen beobachtet. Wenn auch das Wachstum der so behandelten Pflanzen schlecht war und sie nach einiger Zeit eingingen, so waren doch in Langtag nach 4 bis 7 Wochen alle so lange überlebenden Exemplare zur Blütenbildung übergegangen, in Kurztag keines. Die Pfropfungen brachten unter diesen verschärften Bedingungen ein eindeutiges Ergebnis (Tab. 2): bei 18 auswertbaren Exemplaren mit nicht-induziertem Spender keine Blütenbildung, bei 21 mit induziertem Spender Blütenbildung in 20 Fällen. Danach kann gesagt werden, daß Nicotiana tabacum Maryland-Mammut als Spender an einjährigem Hyoscyamus niger als Empfänger Blütenbildung im Pfropfversuch nur dann auslöst, wenn er selbst blühfähig ist.

\section{b) Nicotiana silvestris als Empfänger.}

Mit Nicotiana silvestris als Empfänger konnte nur ein Vorversuch ausgeführt werden. Als Pfropfreiser dienten einzelne Blätter von Maryland-Mammut; die Nicotiana-silvestris-Unterlagen wurden entblättert und die ganzen Pfropfungen in Langtag oder Kurztag gehalten. Bei Nicotiana silvestris führt Entblätterung im Gegensatz zu Hyoscyamus nicht zur Auslösung der Blütenbildung; dies wurde an 12 entblätterten, aber ungepfropften Exemplaren, die bis zum Schluß des Versuches regetativ blieben, bestätigt. Von 10 in
Kurztag gehaltenen Pfropfungen war in 80 Tagen eine zur Blüte gekommen, während bei einer weiteren der Vegetationspunkt stark vergrößert war, Anlage von Blütenprimordien also offensichtlich kurz bevorstand. Von 12 in Langtag gehaltenen Pfropfungen waren in der gleichen Zeit 4 zur Blütenbildung übergegangen.

Sieht man von dem im ganzen unbefriedigenden Ausfall des Versuches ab, so scheint es, daß MarylandMammut als Spender an Nicotiana silvestris als Empfänger Blütenbildung auslösen kann, gleichgültig ob die Maryland-Mammut-Pflanzen selbst blühfähig waren oder nicht. Leider ist die Situation nicht so eindeutig. Entblätterte Nicotiana-silvestris-Pflanzen bleiben zwar längere Zeit am Leben, der Neuzuwachs ist aber im Gegensatz zu gleichartig behandelten HyoscyamusPflanzen gering, und Blütenbildung tritt auch unter Langtagbedingungen nicht ein. Da die Pflanzen kein ausgespochenes Speicherorgan besitzen, und da bis zum Erscheinen von Blütenanlagen bei der Art wesentlich mehr Zeit vergeht als bei Hyoscyamus, so bleibt bei Entblätterung die Blütenbildung vielleicht nur deswegen aus, weil die Pflanzen die notwendigen Assimilate nicht aufzubringen vermögen. Durch die Aufpfropfung eines Maryland-Mammut-Blattes mag dieser Zustand behoben werden; Eintreten von Blütenbildung nach solcher Pfropfung braucht mithin nicht auf einem wirkungsspezifischen Einfluß des Reises zu beruhen. Da sich diese Möglichkeit im Experiment nicht einwandfrei ausschließen läßt, muß die Frage offen bleiben; angesichts des Ergebnisses der Pfropfungen mit Hyoscyamus niger ist im Falle der Nicotiana-silvestris-Empfänger ein unspezifischer Einfluß des Pfropfpartners wahrscheinlicher als ein spezifischer.

\section{Schlußbemerkungen}

Kurztagpflanzen als Empfänger werden im Pfropfversuch - ebenso wie durch Tagneutrale durch blühfähige Langtagpflanzen zur Blütenbildung veranlaßt ${ }^{1-}$. Nach den hier mitgeteilten Ergebnissen ist dies auch in der sinngemäßen reziproken Kombination der Fall. Danach ist es sicher, daß der für die Auslösung der Blütenbildung maßgebende „Endzustand“ bei den beiden photoperiodischen Reaktionstypen und ebenso bei tagneutralen Pflanzen der gleiche ist (oder dal zum mindesten keine tageslängenbedingten Differenzen zwischen ihren „Endzuständen“ bestehen). Über die Natur dieses Endzustandes und die Vorgänge, die zu seiner Erreichung führen, bringen die Versuche nichts Neues. Da diese Fragen soeben von uns an anderer Stelle ${ }^{8}$ eingehend erörtert wurden, brauchen sie hier nicht nochmals aufgegriffen zu werden.

Hingewiesen sei noch auf den unterschiedlichen Ausfall der Pfropfungen mit Hyoscyamus niger 
der Jahre 1942 und 1943. Der verschiedene Anteil der eindeutig unter dem Einfluß des Spenders zur Blütenbildung gekommenen Empfängerpflanzen dürfte auf die verschieden weite Entblätterung der letzten zurückzuführen sein. Durch in Kurztag befindliche Blätter wird der Erfolg der photoperiodischen Induktion einer Langtagpflanze beeinträchtigt ${ }^{11}$; bei den stärker entblätterten Pflan-

11 A. P. Withrow, R. B. With row u. J. P. B i e be l, Plant Physiol. 18, 294 [1943]. zen von 1943 konnte diese Hemmwirkung unter dem Einfluß des blühfähigen Reises leichter überwunden werden. Zwischen der Hemmung der Blütenbildung durch die Blätter unter nicht-induktiven Tageslängenbedingungen und dem auslösenden Einfluß von induzierten Teilen der Pflanze oder von blühfähigen Pfropf reisern besteht offenbar ein Gleichgewicht, und es hängt von der Stärke der beiden Partner ab, nach welcher Seite es verschoben ist.

\title{
Zur Frage der Stoffaufnahme durch die lebende pflanzliche Zelle
}

\author{
V. Zur Theorie der Aufnahme basischer Stoffe \\ Von Horst Drawert \\ Aus dem Botanischen Institut der Universität Jena \\ (Z. Naturforschg. 3b, 111-120 [1948]; eingegangen am 12. Februar 1948)
}

\begin{abstract}
An Hand der geschilderten Versuchsergebnisse wird der Aufnahme basischer Stoffe (Kationenaufnahme) durch die lebende pflanzliche Zelle folgende Arbeitshypothese zugrunde gelegt: Zellsaft und Außenmedium stellen ein Zweiphasensystem dar, in das der Protoplast als Diaphragma eingeschaltet ist. Über die Aufnahme oder Nichtaufnahme eines Stoffes in die Vakuole entscheidet in erster Linie der Koeffizient seiner Verteilung zwischen Außenmedium und Zellsaft, der durch folgende Faktoren bestimmt wird: H-Ionen-Konzentrations-Gefälle Außenmedium/Zellsaft, Dissoziationsgrad des aufzunehmenden Stoffes, Gehalt des Außenmediums und des Zellsaftes an adsorbierenden Substanzen wie Kolloiden und sauren Lipoiden. Die Permeabilität hat erst in zweiter Linie für die Stoffaufnahme Bedeutung als begrenzender Faktor im Sinne des Ultrafilterprinzips. Für die Aufnahme eines basischen Stoffes in das Protoplasma gibt dagegen der Verteilungskoeffizient, der auf einer verschiedenen Löslichkeit des Stoffes in hydrophilen und hydrophoben Medien berubt, den Ausschlag. Die die Stoffaufnahme beeinflussenden Faktoren, wie Wasserstoffionenkonzentration, Salze, Licht, Temperatur u.a., wirken weniger verändernd auf die Permeabilität des Cytoplasmas - wie bisher allgemein angenommen -, sondern vor allem auf die Verteilung des untersuchten Stoffes zwischen Außenmedium und Zellsaft, indem sie eine Verschiebung des Dissoziationsgrades der aufzunehmenden Verbindung bedingen und über den Stoffwechsel den $p_{\mathrm{H}}$-Wert des Zellsaftes ändern. - Das lebende Cytoplasma wird als sehr stabiler, nach außen reaktionsträger Eiweiß-Lipoid-Komplex aufgefaßt, dessen Carboxyl- und Aminogruppen durch Lipoide abgeschirmt sind, und das in seinen Eigenschaften einem neutralen, hydrophoben Lipoid entspricht. Erst beim Absterben wird die Eiweiß-Lipoid-Bindung gesprengt und das Cytoplasma auch nach außen reaktionsfähig.
\end{abstract}

$\mathrm{D}$ e heute in der Pflanzenphysiologie vorherrschende Ansicht über die Stoffaufnahme durch die lebende Zelle sieht in den Permeabilitätseigenschaften der Plasmagrenzflächen den diesen Vorgang in erster Linie bestimmenden Faktor. Die Zusammensetzung der Lipoide in den Grenzflächen, Größe und Anzahl der Poren im Plasmalemma und im Tonoplasten, oder auf Ionenaustausch beruhende Adsorptionsvorgänge an den Grenzflächen des Protoplasten werden für die
Permeabilität und damit für die Stoffaufnahme verantwortlich gemacht. Die Identität von Stoffaufnahmegeschwindigkeit und Permeiervermögen ist im pflanzenphysiologischen Denken seit Jahrzehnten so fest verankert, daß man aus der Stoffaufnahmegeschwindigkeit auf erblich fixierte Permeabilitätseigenschaften der Plasmagrenzflächen schließt und auf ,spezifische Permeabilitätsreihen" eine ,vergleichende Protoplasmatik“ begründet hat (Höfler). Darauf aufbauend, ver- 